\title{
An Optimal Inventory Policy for Subsequent Price Reduction Problem
}

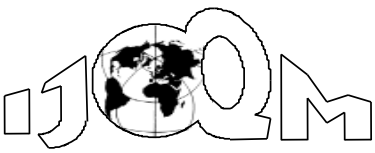

DOI: $10.46970 / 2020.26 .2 .4$

Volume 26, Number 2

June 2020, pp. 147-161

\author{
Suresha Kharvi \\ T. P.M. Pakkala \\ Mangalore University \\ (kharvisuresh@gmail.com) \\ (tpm.pakkala@gmail.com)
}

This article considers an optimal inventory policy of the retailer in response to a special price strategy on subsequent orders by the supplier. A finite horizon model with constant yearly demand is considered in this article. The closed form solution for optimal order quantities and an optimal number of orders is obtained by minimizing the total cost. Numerical and sensitivity analysis are carried out to support the theoretical results. A significant percentage difference in cost between the developed optimal policy and the EOQ policy highlights the importance of the developed optimal policy.

Keywords: Inventory, Two-Purchase Price, Three-Purchase Price, Optimal Number of Orders, Optimal Order Quantity, Optimal Cost.

\section{Introduction and Literature Review}

A supplier cannot build a successful business when the retailer orders items once and never orders again. For a supplier, it is important that a retailer frequently purchases from him. Hence, for the supplier, it is necessary to design a pricing strategy, which encourages the retailer to continue ordering from him. One such pricing strategy is to reasonably reduce the purchase price of the items after the first purchase. Once a new retailer purchases items with regular price, the supplier offers a price reduction from next purchase onwards in order to retain the retailer. This kind of reward to a frequent buyer is common in today's business through the loyalty programme. A loyalty programme is a strategy designed for buyers to continue to purchase items. As an example of this is the Target RED Card, which rewards its buyers 5\% reduction in purchase price every time they purchase. Big Lots offers $\$ 5$ off for subsequent purchase when the buyer joins the Big Rewards customer loyalty programme. By implementing this kind of pricing strategy, a supplier attracts more retailers and hence, can generate more revenue. On the other hand, the retailer gets the benefit of the reduced purchase price.

The present paper considers the ordering policy of a retailer when the supplier reduces the purchase price of the items after the first purchase. This model is extended in which the supplier further gives discount third order onwards. These discounts are already known in advance. The yearly demand of the customers is assumed to be constant and known to the retailer. In such a scenario, the retailer has to decide how much to order, so that the total cost of the inventory is minimum. In the first model, the pricing strategy of the supplier is as follows: 
For the first purchase, the purchase price of the item is $P$. Let $P \alpha,(0<\alpha<1)$, be the purchase price of the item for the second purchase onwards.

Corresponding to this pricing strategy, the retailer has to develop an optimal ordering policy, which is the objective of the present article. This kind of pricing strategy not only motivates the retailer to buy next time, but also attracts new retailers. This pricing strategy is a two-price inventory problem. Many researchers have shown interest in price change problems. Most of the models assume one price over an interval of time and reduced price in another interval of duration. In these durations, there may be multiple orders, unlike the situation discussed here. The price change inventory problem was first studied by Naddor (1966), who proposed the infinite horizon EOQ model with the assumption that price change occurs at the end of an EOQ cycle. Goyal (1975) studied the EOQ model when the price changes followed a straight-line rule. The optimal ordering policy when the price changes continuously due to inflation was studied by Buzacott (1975) and Erel (1992). Lev and Soyster (1979) developed a finite horizon inventory model and determined the optimal ordering policies when information about an ensuing price rise was known. An improved algorithm for determining the optimal policy for the model of Lev and Soyster (1979) was provided by Goyal (1980). Taylor and Bradley (1985) extended Naddor's (1966) model and found optimal ordering strategies for situations where the price increase did not coincide with the end of the EOQ cycle. Lev and Wiess (1990) also developed a comprehensive model that covered both finite and infinite horizons under purchase price increase and decrease. Their algorithm for solving the finite horizon EOQ model with price change was modified by Andre Gascon (1995). Ghosh (2003) and Huang and Kulkarni (2003) discussed the infinite horizon deterministic inventory model under an announced price increase. Khouja and Park (2003) developed a closed form approximate solution for an inventory model with continuous decrease in purchase price. They made a restrictive assumption of the equal cycle time. Their model was improved by Khouja and Goyal (2006) by considering cycle times of varying lengths. Arcelus et al. (2006) modelled the retailer's response to special sales characterized by uncertain termination dates, but with the ending date known at the time of its realization. An EOQ model with known price increase and partial backordering was developed by Taleizadeh and Pentico (2013). Pakkala and Babitha (2013) developed an optimal inventory policy for the two- price problem for a situation in which the discount price is announced and will remain for a random duration.

Even though there are many articles on price change problem, as far as is known no attempt has been made to develop a model for subsequent price reduction by the supplier. The solution discussed in the earlier literature of price change is different as the price change occurs after a certain point of time, which may be known or uncertain, and hence, the price change is time dependent. But the present article considers the price change problem, where the price change occurs after the first purchase, and after the second purchase, and hence, the discount is not directly time dependent. This leads to the derivation of a new solution procedure for the problem mentioned here.

The paper is organized as follows. Assumptions and notations used in the article is considered in Section 2. Section 3 presents the model development. In Section 4, the two- price model is extended to three-price problem. Numerical and sensitivity analysis is considered in Section 5. Section 6 presents the comparison of the optimal policy with the EOQ policy. Section 7 concludes the paper. 


\section{Assumptions and Notation}

First, the two- price problem is discussed, that is, the situation of price discount is offered after the first order. The following assumptions and notations are used in the article.

\section{Assumptions}

1. Planning horizon is finite and known;

2. Demand Is Constant and Uniform Over the Planning Horizon;

3. Shortages are not allowed;

4. Initial inventory is zero; and

5. Lead time is zero.

\section{Notation}

1. $P$ is the actual purchase price of the item,

2. $T$ is the planning horizon (in number of years),

3. $\alpha$ is the discount rate on the purchase price for first purchase onwards of the retailer,

4. $D$ is the demand for one year,

5. $K$ is the ordering cost,

6. $F$ is the carrying cost fraction per unit amount of money per year,

7. $m$ is the number of orders after the first purchase, and

8. $n$ is the total number of orders throughout the planning horizon.

\section{Model Development}

Let $Q^{\prime}$ be the order quantity corresponding to the first purchase. Let $Q$ be the quantity of items ordered by the retailer from the first purchase onwards. Let $t_{1}, t_{2}, t_{3}, \ldots, t_{n}$ be the time points at which $n$ orders are placed. The inventory position is represented in Figure 1.

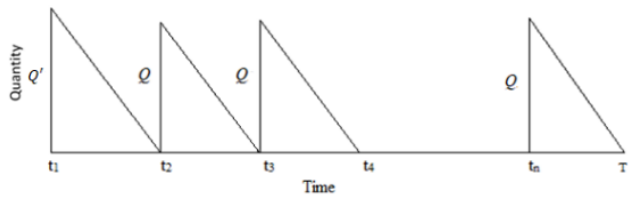

Figure 1 Inventory Position

Since the total number of orders is $m+1$ with the order cost of $K$ per order, the total set-up cost is $(m+1) K$. The total items' cost over the planning horizon $T$ is $Q^{\prime} P+$ $m Q P \alpha$, since $Q$ 'and $Q$ ( $m$ times) items are ordered at price $P$ and $P \alpha$, respectively. The average inventory for the first order cycle is $Q^{\prime} / 2$, which is kept for the time of $Q^{\prime} / D$, hence, the holding cost for this cycle is $\frac{Q^{\prime 2} P F}{2 D}$. Similarly, the holding cost for the remaining order cycles is given by $\frac{m Q^{2} P \alpha F}{2 D}$. The different costs of the model are listed in the following Table. 
Table 1 Various costs of the Inventory Model

\begin{tabular}{|l|c|}
\hline Type of the cost & Cost \\
\hline Total set-up cost & $(m+1) K$ \\
\hline Items' cost & $Q^{\prime} P+m Q P \alpha$ \\
\hline Holding cost & $\frac{Q^{\prime 2} P F}{2 D}+\frac{m Q^{2} P \alpha F}{2 D}$ \\
\hline
\end{tabular}

Hence, the total cost is given by

$T C=(m+1) K+Q^{\prime} P+m Q P \alpha+\frac{{Q^{\prime}}^{2} P F}{2 D}+\frac{m Q^{2} P \alpha F}{2 D}$

Since the yearly demand is $D$, we have $Q^{\prime}+m Q=T D$. Hence, $Q^{\prime}$ can be written as $Q^{\prime}=T D-m Q$.

Therefore, the total cost can be written as function $C_{m}($ ) of a single variable $Q$.

$C_{m}(Q)=(m+1) K+(T D-m Q) P+m Q P \alpha+\frac{(T D-m Q)^{2} P F}{2 D}+\frac{m Q^{2} P \alpha F}{2 D}$

The optimal value of order quantity $Q$ for a given value of $m$ is derived in the following theorem.

\section{Theorem 1}

The optimal order quantity corresponding to purchase price $P \alpha$ for a given value of $m$ is given by

$Q_{m}^{*}=\frac{D}{F}\left(\frac{1-\alpha+T F}{m+\alpha}\right)$

\section{Proof}

We have $C_{m}(Q)=(m+1) K+(T D-m Q) P+m Q P \alpha+\frac{(T D-m Q)^{2} P F}{2 D}+\frac{m Q^{2} P \alpha F}{2 D}$

$C_{m}(Q)=(m+1) K+T D P+\frac{P F T^{2} D}{2}-m Q P(1-\alpha+F T)+\frac{m Q^{2} P F(m+\alpha)}{2 D}$

After equating the first derivative of $C_{m}$ with respect to $Q$ to zero yields

$\frac{d C_{m}}{d Q}=-m P(1-\alpha+F T)+\frac{m Q P F(m+\alpha)}{D}=0$,

which implies that $Q_{m}^{*}=\frac{D}{F}\left(\frac{1-\alpha+T F}{m+\alpha}\right)$.

It can be observed that the optimal order quantity depends on the number of orders $m$, which is also a decision variable. After the substitution of $Q_{m}^{*}$ in (2), the optimal cost for a given $m$, denoted by $C^{*}(m)$, can be found andis given by 


$$
C^{*}(m)=(m+1) K+T D P+\frac{P F T^{2} D}{2}-\frac{m P F L^{2}}{2 D(m+\alpha)}
$$

Where $L=\frac{D}{F}(1-\alpha+T F)$.

It can be noted that $C^{*}(m)$ is independent of optimal $Q^{\prime}$ and optimal $Q$.This is because optimal $Q$ depends on $m$ and other parameters, and $Q^{\prime}$ is $T D-m Q$ expressed in terms of $m$ and parameters only. The optimal order quantity corresponding to purchase price $P \alpha$ and the optimal cost of the model can be found by obtaining optimal number of orders after the first purchase, denoted by $m^{*}$, which minimizes (4). Hence, the determination of the optimal number of orders requires special attention. The derivation of the optimal number of orders is based on the fact that function $C^{*}($ )is a convex function of $m$, which is proved in the following theorem.

\section{Theorem 2}

Function $C^{*}$ is a discrete convex function of $m$.

\section{Proof}

The function $C^{*}$ is the convex function of $m$, if $C^{*}(m-1)+C^{*}(m+1)>2 C^{*}(m)$, for all $m \geq 1$.

Let $m(\geq 1)$ be a arbitrary number . Assume $C^{*}(m-1)+C^{*}(m+1)-2 C^{*}(m)>0$.

Which implies, $-\frac{(m-1)}{(m-1+\alpha)}-\frac{(m+1)}{(m+1+\alpha)}+\frac{2 m}{(m+\alpha)}>0$.

After simplification, the above inequality yields $\alpha>0$, which is always true.

Hence, (5) holds for all $m \geq 1$.

\subsection{Determination of Optimal Number of orders after the first order}

The optimal number of orders after the first purchase can be found by the search method. But if it is very large, it may be difficult to find the optimal $m$ through the search procedure. In the following theorem, theclosed form solution to the optimal number of orders after the purchase is derived

\section{Theorem 3}

The optimal number of orders after the first purchase, denoted by $m^{*}$, is given by

$m^{*}=\left[\frac{1}{2}+\sqrt{\frac{P F L^{2} \alpha}{2 D K}+\frac{1}{4}}-\alpha\right]$.

Where, $[x]$ is the largest integer less than or equal to $x$.

\section{Proof}

Function $C^{*}$ can be written as $C^{*}(m)=(m+1) K+C_{0}-\frac{m C^{\prime}}{(m+\alpha)}$, where $C_{0}=T D P+$ $\frac{P F T^{2} D}{2}, C^{\prime}=\frac{P F L^{2}}{2 D}$. 
Since $C^{*}$ is a convex function of $m$, there existsm* such that $C^{*}\left(m^{*}\right) \leq C^{*}\left(m^{*}-1\right)$ and $C^{*}\left(m^{*}\right) \leq C^{*}\left(m^{*}+1\right)$.

This $m^{*}$ will be the optimal number of orders after the first purchase. It can also be seen that $m^{*}=\max \left\{m \geq 1: C^{*}(m) \leq C^{*}(m-1)\right\}$.

Consider $C^{*}(m) \leq C^{*}(m-1)$

$\Rightarrow(m+1) K+C_{0}-\frac{m C^{\prime}}{(m+\alpha)} \leq m K+C_{0}-\frac{(m-1) C^{\prime}}{(m-1+\alpha)}$

$\Rightarrow m^{\prime}\left(m^{\prime}-1\right) \leq \frac{c^{\prime} \alpha}{K}$, where $m^{\prime}=(m+\alpha)$

$\Rightarrow m^{\prime 2}-m^{\prime}-\frac{c^{\prime} \alpha}{K} \leq 0$.

First let us find the value of $m$ (need not be an integer), which satisfies $\mathrm{m}^{\prime 2}-\mathrm{m}^{\prime}-\frac{\mathrm{C}^{\prime} \alpha}{\mathrm{K}}=0$.

The feasible solution of mis $m=\frac{1}{2}+\sqrt{\frac{P F L^{2} \alpha}{2 D K}+\frac{1}{4}}-\alpha$. Hence, for $m=\frac{1}{2}+\sqrt{\frac{P F L^{2} \alpha}{2 D K}+\frac{1}{4}}-\alpha \quad$ we $\quad$ have $\quad m^{\prime 2}-m^{\prime}-\frac{C^{\prime} \alpha}{K}=0$. Therefore, for $m=\left[\frac{1}{2}+\sqrt{\frac{P F L^{2} \alpha}{2 D K}+\frac{1}{4}}-\alpha\right] \leq \frac{1}{2}+\sqrt{\frac{P F L^{2} \alpha}{2 D K}+\frac{1}{4}}$, we have $(m+\alpha)^{2}-(m+\alpha)-$ $\frac{C^{\prime} \alpha}{K} \leq 0$.

It can be observed that $m=\left[\frac{1}{2}+\sqrt{\frac{P F L^{2} \alpha}{2 D K}+\frac{1}{4}}-\alpha\right]$ is maximum among the integers, which satisfies $m^{\prime 2}-m^{\prime}-\frac{c^{\prime} \alpha}{K} \leq 0$. Hence, the optimal number of orders $m^{*}=\left[\frac{1}{2}+\sqrt{\frac{P F L^{2} \alpha}{2 D K}+\frac{1}{4}}-\alpha\right]$.

It may be noted that for a single price in a finite horizon problem, the closed form solution to the number of orders is given by Lev and Weiss (1990) and Arcelus et al. (2015).

The algorithm to obtain the optimal number of orders, optimal order quantities, and optimal cost when $m \geq 1$ is given below, however, $m=0$ could be optimal in rare cases, especially when the finite horizon is small. In such cases, the cost for a single order for the entire planning horizon with price $P$ is also computed and compared to determine optimality.

\subsection{Algorithm to Obtain Optimal Number of Orders, Optimal Order Quantities, and Optimal Cost}

The following steps are used to obtain the optimal number of orders, optimal order quantities and optimal cost. 


\section{Step 1}

Compute $m^{*}=\left[\frac{1}{2}+\sqrt{\frac{P F L^{2} \alpha}{2 D K}+\frac{1}{4}}-\alpha\right]$. This will be the optimal number of orders after the first purchase.

\section{Step 2}

Compute $\frac{D}{F}\left(\frac{1-\alpha+T F}{m^{*}+\alpha}\right)$, this gives the optimal quantity, $Q^{*}$ to be ordered after the first purchase.

\section{Step 3}

Obtain an optimal order quantity corresponding to first purchase $\mathrm{Q}^{\prime^{*}}$ using thefomula $Q^{\prime *}=T D-m^{*} Q^{*}$.

\section{Step 4}

Optimal cost can be obtained by substituting $m^{*}=\left[\frac{1}{2}+\sqrt{\frac{P F L^{2} \alpha}{2 D K}+\frac{1}{4}}-\alpha\right]$ in (4).

\section{Extension of the Model to Three Prices}

The above two- price model can be extended to the three- price problem. One of the pricing strategies, which leads to the three- price inventory problem is considered below.

For the first purchase, the purchase price is $P$. Let $P \alpha_{1},\left(0<\alpha_{1}<1\right)$, be the purchase price of the item for the second purchase. For $2^{\text {nd }}$ purchase onwards, let $P \alpha_{2},\left(0<\alpha_{2}<\right.$ $\left.1, \alpha_{2}<\alpha_{1}\right)$ be the purchase price. Let $Q_{1}$ and $Q_{2}$ be the order quantity corresponding to the first and second purchase. Let $Q_{3}$ be the quantity of items ordered by the retailer from the $2^{\text {nd }}$ purchase onwards. Let $m$ be the number of orders after the second purchase (note that for two- price problem, $m$ is the number of orders after the first purchase). The total cost for the planning horizon Tis given by

$C_{m}\left(Q_{1}, Q_{2}, Q_{3}\right)=(m+2) K+Q_{1} P+Q_{2} P \alpha_{1}+m Q_{3} P \alpha_{2}+\frac{Q_{1}^{2} P F}{2 D}+\frac{Q_{2}^{2} P \alpha_{1} F}{2 D}+$ $\frac{m Q_{3}^{2} P \alpha_{2} F}{2 D}$

Since the yearly demand is $D$, we have $Q_{1}+Q_{2}+m Q_{3}=T D$.

The optimal values of $Q_{1}, Q_{2}$, and $Q_{3}$ for a given value of $m$ is derived in the following theorem.

\section{Theorem 4}

The optimal order quantities for a given value of $m$ is given by:

$Q_{1}^{*}=\frac{D}{F}\left(\left(\frac{T F+m+2}{1+\frac{1}{\alpha_{1}}+\frac{m}{\alpha_{2}}}\right)-1\right), Q_{2}^{*}=\frac{D}{F}\left(\left(\frac{T F+m+2}{\alpha_{1}\left(1+\frac{1}{\alpha_{1}}+\frac{m}{\alpha_{2}}\right)}\right)-1\right)$ and 
$Q_{3}^{*}=\frac{D}{F}\left(\left(\frac{T F+m+2}{\alpha_{2}\left(1+\frac{1}{\alpha_{1}}+\frac{m}{\alpha_{2}}\right)}\right)-1\right)$

\section{Proof}

We use Lagrange's multiplier technique to minimize $C_{m}\left(Q_{1}, Q_{2}, Q_{3}\right)$ subject to the constraint $Q_{1}+Q_{2}+m Q_{3}=T D$.

Lagrange's multiplier function is given by

$$
\begin{aligned}
& L_{m}\left(Q_{1}, Q_{2}, Q_{3}\right)=(m+2) K+Q_{1} P+Q_{2} P \alpha_{1}+m Q_{3} P \alpha_{2}+\frac{Q_{1}^{2} P F}{2 D}+\frac{Q_{2}^{2} P \alpha_{1} F}{2 D}+ \\
& m \frac{Q_{3}^{2} P \alpha_{2} F}{2 D}-\lambda\left(g_{m}\left(Q_{1}, Q_{2}, Q_{3}\right)-T D\right)
\end{aligned}
$$

Where, $g_{m}\left(Q_{1}, Q_{2}, Q_{3}\right)=Q_{1}+Q_{2}+m Q_{3}$ and $\lambda$ is Lagrange's multiplier.

Equating the partial derivative of Lagrange's function with respect to each variable and Lagrange's multiplier to zero yields the following set of equations

$\frac{\partial L_{m}}{\partial Q_{1}}=P+\frac{Q_{1} P F}{D}-\lambda=0$

$\frac{\partial L_{m}}{\partial Q_{2}}=P \alpha_{1}+\frac{Q_{2} P \alpha_{1} F}{D}-\lambda=0$

$\frac{\partial L_{m}}{\partial Q_{3}}=m P \alpha_{2}+\frac{m Q_{3} P \alpha_{2} F}{D}-m \lambda=0$

$Q_{1}+Q_{2}+m Q_{3}-T D=0$

Solving the first three equations yields

$Q_{1}=\frac{D}{P F}(\lambda-P), Q_{2}=\frac{D}{P \alpha_{1} F}\left(\lambda-P \alpha_{1}\right)$, and $Q_{3}=\frac{D}{P \alpha_{2} F}\left(\lambda-P \alpha_{2}\right)$.

Substituting these values in (12), we get $\lambda=\left(\frac{T F+m+2}{1+\frac{1}{\alpha_{1}}+\frac{m}{\alpha_{2}}}\right) P$.

Hence, we have $Q_{1}=\frac{D}{F}\left(\left(\frac{T F+m+2}{1+\frac{1}{\alpha_{1}}+\frac{m}{\alpha_{2}}}\right)-1\right), Q_{2}=\frac{D}{F}\left(\left(\frac{T F+m+2}{\alpha_{1}\left(1+\frac{1}{\alpha_{1}}+\frac{m}{\alpha_{2}}\right)}\right)-1\right)$ and

$Q_{3}=\frac{D}{F}\left(\left(\frac{T F+m+2}{\alpha_{2}\left(1+\frac{1}{\alpha_{1}}+\frac{m}{\alpha_{2}}\right)}\right)-1\right)$

In order to prove that the cost function $C_{m}$ ( ) attains its minimum at these stationary points, one needs to prove that the determinant of the borded Hessian matrix at these stationary points is negative. The borded Hessian matrix, denoted by $H$,is given by $H=\left[\begin{array}{ccc}0 & 1 & 1 \\ 1 & 1 & 0 \\ 1 & 0 & \alpha_{1}\end{array}\right]$ 
The determinant of this matrix is $-1-\alpha_{1}$ which is always negative.

Hence, $Q_{1}=\frac{D}{F}\left(\left(\frac{T F+m+2}{1+\frac{1}{\alpha_{1}}+\frac{m}{\alpha_{2}}}\right)-1\right), Q_{2}=\frac{D}{F}\left(\left(\frac{T F+m+2}{\alpha_{1}\left(1+\frac{1}{\alpha_{1}}+\frac{m}{\alpha_{2}}\right)}\right)-1\right)$ and

$Q_{3}=\frac{D}{F}\left(\left(\frac{T F+m+2}{\alpha_{2}\left(1+\frac{1}{\alpha_{1}}+\frac{m}{\alpha_{2}}\right)}\right)-1\right)$

will minimize the cost function $C_{m}\left(Q_{1}, Q_{2}, Q_{3}\right)$ for a given value of $m$.After the substitution of optimal values of $Q_{1}, Q_{2}$, and $Q_{3}$, the cost function becomes the function of the single variable $m$. This function is denoted by $C^{*}()$, which is given by

$C^{*}(m)=(m+2) K+\frac{P D}{F} \emptyset_{1}(m)+\frac{P D}{2 F} \emptyset_{2}(m)$

Where $\emptyset_{1}(m)=\Psi(m)(m+2)-\left(1+\alpha_{1}+m \alpha_{2}\right)$,

$\emptyset_{2}(m)=(\Psi(m)-1)^{2}+\frac{\left(\Psi(m)-\alpha_{1}\right)^{2}}{\alpha_{1}}+m \frac{\left(\Psi(m)-\alpha_{2}\right)^{2}}{\alpha_{2}} ; \Psi(m)=\frac{T F+m+2}{1+\frac{1}{\alpha_{1}}+\frac{m}{\alpha_{2}}}$.

It can be proved that $C^{*}$ is a discrete convex function of $m$ (the proof is similar to that of the two- price case). It is difficult to find the closed form solution for optimalm value, since it involves a complicated expression. We use the following algorithm to find the optimal number of orders after the second purchase. Once the optimal number of orders is found, the optimal order quantities and the optimal cost can be found. The optimal number of orders $n=\left[\sqrt{\frac{\mathrm{DPFT}^{2} \alpha}{2 \mathrm{~K}}+\frac{1}{4}}-\frac{1}{2}\right]$ for single price (finite horizon) case is considered as an initial value for $n(=m+2)$. Where, [ ]indicates upper integer part. Hence, the initial value for $m$ will be $m=\left[\sqrt{\frac{\text { DPFT }^{2} \alpha}{2 \mathrm{~K}}+\frac{1}{4}}-\frac{1}{2}\right]-2$. This may not be optimal $m$, but close to it. When the planning horizon is small, we need to compare the optimal cost corresponding to a single order with price $P$ alone for the entire planning horizon and two- order with prices $P$ and $P \alpha_{1}$ for optimality. In both these cases, $m=0$.

\subsection{Algorithm to Find Optimal Number of Orders}

\section{Step 1}

Compute $m=\left[\sqrt{\frac{D P F T^{2} \alpha}{2 K}+\frac{1}{4}}-\frac{1}{2}\right]-2$. Check whether this $m$ satisfies the condition $C^{*}(m-1)>C^{*}(m)$ and $C^{*}(m)<C^{*}(m+1)$. 
If it satisfies condition (15), then the optimal number of orders after the second purchase will be $\left[\sqrt{\frac{D P F T^{2} \alpha}{2 K}+\frac{1}{4}}-\frac{1}{2}\right]-2$.If this $m$ does not satisfy condition (15), then it will satisfy either one of the following conditions

$$
\begin{aligned}
& \text { 1. } C^{*}(m+1)<C^{*}(m)<C^{*}(m-1) \\
& \text { 2. } C^{*}(m-1)<C^{*}(m)<C^{*}(m+1)
\end{aligned}
$$

\section{Step 2}

\section{Case 1}

If (16) is satisfied by $m$ in Step 1, then this $m$ will be less than the optimal $m$. Hence, the $m$ value of Step 1 has to be increased by 1 and has to verify condition (15) again for $m+1$. This process will continue until we arrive at optimal $m$, which satisfies (15). This process will terminate quickly.

\section{Case 2}

If (17) is satisfied by $m$ in Step 1, then this $m$ will be greater than optimal $m$. Hence, the $m$ value of Step 1 has to be decreased by 1 and has to verify condition (15) again form -1 . This process will continue until we arrive at optimal $m$, which satisfies (15).

\section{Step 3}

Once the optimal value of $m$ (denoted by $m *$ ) is determined, the optimal order quantities can be found by substituting this optimal value of $m$ in (13). By substituting the optimal order quantities in (7), the optimal cost can be found. When $\alpha_{1}=1$ and $\alpha_{1}=$ $\alpha_{2}$, the three-price problem reduces to the two- price problem.

\section{Numerical and Sensitivity Analysis}

To support the theoretical results, a numerical illustration was considered. Suppose a retailer orders items from a supplier with the purchase price for the $1^{\text {st }}$ purchase as $P=$ Rs. 100. The set-up cost per order is taken as Rs. 1200. In order to make a new retailer to buy again, the supplier reduces the purchase price by $2 \%\left(\alpha_{1}=0.98\right)$ for the second purchase, and by $5 \%$ fromthe second purchase onwards $\left(\alpha_{2}=0.95\right)$ The yearly demand and planning horizon isconsidered to be 1500 items and 5 years, respectively. The carrying cost fraction per year per Rs is assumed to be 0.4 . These parameters are said to be the basic parameters.

The optimal number of orders is found to be $n^{*}=25$ and hence, $m^{*}=23$. According to the optimal policy, quantity to be ordered for the first purchase and the second purchase is $Q_{1}^{*}=109.9464$ units and $Q_{2}^{*}=188.7208$ units. Then, from the second purchase onwards, the retailer has to order $Q_{3}^{*}=313.1014$ units, whichresults in minimum cost of Rs.772802.6 for the planning horizon of 5 years. For this set of parameters, the convex property of $C^{*}$ with respect to the number of orders $m$ is displayed in Figure 2. 


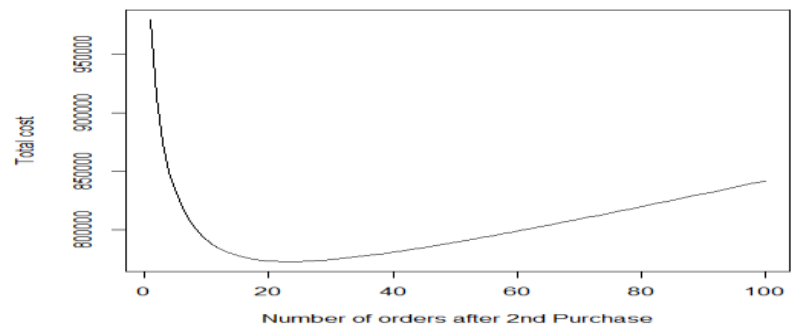

Figure 2 Total cost vs. Number of Orders after Second Purchase

Table 2 and Table 3 show the sensitivity analysis for the three-price and two-price problems, respectively. The first two columns represent the parameters and its value. The parameter values are changed by $-40 \%,-20 \%, 20 \%$, and $40 \%$ with respect to the basic parameters. The basic parameter for the two- price problem is $(K, F, D, T, P, \alpha)$ $=(1200,0.4,1500,5,100,0.95)$.Column 3 of Table 2 represents the optimal number of orders after the second order. Similarly, Column 3 of Table 3 represents the optimal number of orders after the first order. The optimal order quantities are considered in Columns4, 5, and 6 of Table 2 for the three-price problem, and in Columns 4 and 5 of Table 3 for the two-price problem. Column 7 of Table 2 and Column 6of Table 3 give the optimal cost of model $C^{*}$ for the three-price and two-price problems, respectively. The last column of both the Tables gives the percentage change in the optimal cost relative to the optimal cost corresponding to the base parameter. The following observations can be drawn from Table 2 and Table 3.

1. The retailer thinks of ordering less quantity during the first purchase since he can buy more items at a lower price after the first purchase. But when the ordering cost increases, the retailer has to increase the order quantity of the first order, which can be observed from the Table.

2. As expected, the number of orders decreases with the increase in set-up cost.

3. The optimal number of orders increases with the carrying cost fraction. The reason for this is that it is better to have less inventory when the holding cost is more. Hence, the optimal order quantity for each order is less. But in such a scenario, the retailer must order a greater number of times since the total order quantity for the planning horizon is fixed.

4. The purchase price and demand will have the same impact on optimal cost.

Table 2 Sensitivity Analysis for Three-Price Problem

\begin{tabular}{|c|c|c|c|c|c|c|c|}
\hline Parameters & Parameter value & $m^{*}$ & $Q_{1}^{*}$ & $Q_{2}^{*}$ & $Q_{3}^{*}$ & $C^{*}(R s)$. & Percentage Difference \\
\hline$D$ & 900 & 18 & 110.7653 & 158.9442 & 235.0161 & 474341.9 & -38.6206 \\
\hline & 1200 & 21 & 108.7218 & 172.1651 & 272.3387 & 624002.2 & -19.2546 \\
\hline & 1800 & 26 & 93.58211 & 187.3287 & 335.3496 & 920973.2 & 19.1731 \\
\hline & 2100 & 28 & 84.33375 & 193.1977 & 365.0882 & 1068684 & 38.2868 \\
\hline$K$ & 720 & 31 & 37.66157 & 114.9608 & 237.0122 & 759042.8 & -1.7805 \\
\hline & 960 & 26 & 77.98509 & 156.1072 & 279.458 & 766357.6 & -0.8339 \\
\hline & 1440 & 21 & 135.9022 & 215.2064 & 340.4234 & 778622.8 & 0.7531 \\
\hline & 1680 & 20 & 150.6564 & 230.2616 & 355.9541 & 783997.7 & 1.4486 \\
\hline
\end{tabular}




\begin{tabular}{|c|c|c|c|c|c|c|c|}
\hline$F$ & 0.24 & 18 & 69.22033 & 198.184 & 401.8109 & 759873.5 & -1.6730 \\
\hline & 0.32 & 21 & 92.15974 & 189.7038 & 343.7208 & 766737.7 & -0.7848 \\
\hline & 0.48 & 26 & 107.5207 & 173.4906 & 277.6534 & 778281.5 & 0.7089 \\
\hline & 0.56 & 28 & 111.0674 & 167.9988 & 257.8905 & 783329.1 & 1.3621 \\
\hline$P$ & 60 & 18 & 184.6089 & 264.907 & 391.6936 & 474341.9 & -38.6206 \\
\hline & 80 & 21 & 135.9022 & 215.2064 & 340.4234 & 624002.2 & -19.2546 \\
\hline & 120 & 26 & 77.98509 & 156.1072 & 279.458 & 920973.2 & 19.1731 \\
\hline & 140 & 28 & 60.2384 & 137.9984 & 260.7773 & 1068684 & 38.2868 \\
\hline$T$ & 3 & 14 & 99.08019 & 177.6328 & 301.6634 & 464409 & -39.9057 \\
\hline & 4 & 19 & 98.7025 & 177.2475 & 301.2658 & 618608.8 & -19.9525 \\
\hline & 6 & 28 & 107.8664 & 186.5983 & 310.912 & 926993.5 & 19.9522 \\
\hline & 7 & 33 & 106.382 & 185.0837 & 309.3495 & 1081187 & 39.9047 \\
\hline
\end{tabular}

Table 3 Sensitivity Analysis for Two-Price

\begin{tabular}{|c|c|c|c|c|c|c|}
\hline Parameters & $\begin{array}{c}\text { Parameter } \\
\text { Value } \\
\end{array}$ & $m$ & $Q^{\prime^{*}}$ & $Q^{*}$ & $C^{*}(R s)$. & $\begin{array}{l}\text { Percentage } \\
\text { Difference }\end{array}$ \\
\hline \multirow[t]{4}{*}{$D$} & 900 & 18 & 118.7335 & 243.4037 & 473720.2 & -38.6400 \\
\hline & 1200 & 21 & 116.1731 & 280.1822 & 623307.7 & -19.2642 \\
\hline & 1800 & 26 & 100.1855 & 342.3006 & 920169.0 & 19.1876 \\
\hline & 2100 & 29 & 78.8815 & 359.3489 & 1067835.0 & 38.3145 \\
\hline \multirow[t]{4}{*}{$K$} & 720 & 31 & 41.0798 & 240.6103 & 758500.7 & -1.7529 \\
\hline & 960 & 27 & 73.7925 & 275.0447 & 765693.7 & -0.8212 \\
\hline & 1440 & 22 & 130.7190 & 334.9673 & 777768.7 & 0.7428 \\
\hline & 1680 & 20 & 161.0979 & 366.9451 & 783042.5 & 1.4259 \\
\hline \multirow[t]{4}{*}{$F$} & 0.24 & 19 & 59.5238 & 391.604 & 758970.2 & -1.6921 \\
\hline & 0.32 & 22 & 85.7843 & 337.0098 & 765927.2 & -0.7910 \\
\hline & 0.48 & 26 & 113.6364 & 284.0909 & 777570.5 & 0.7171 \\
\hline & 0.56 & 28 & 116.5803 & 263.6936 & 782662.7 & 1.3767 \\
\hline \multirow[t]{4}{*}{$P$} & 60 & 18 & \begin{tabular}{|l|}
197.8892 \\
\end{tabular} & 405.6728 & 473720.2 & -38.6400 \\
\hline & 80 & 21 & 145.2164 & 350.2278 & 623307.7 & -19.2642 \\
\hline & 120 & 26 & 83.4879 & 285.2505 & 920169.0 & 19.1876 \\
\hline & 140 & 29 & \begin{tabular}{|l|}
56.3439 \\
\end{tabular} & 256.6778 & 1067835.0 & 38.3145 \\
\hline \multirow[t]{4}{*}{ 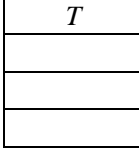 } & 3 & 14 & 110.3679 & 313.5452 & 463648.0 & -39.9446 \\
\hline & 4 & 19 & 107.1429 & 310.1504 & 617839.3 & -19.9725 \\
\hline & 6 & 29 & 103.9232 & 306.7613 & 926230.6 & 19.9727 \\
\hline & 7 & 34 & 103.0043 & 305.7940 & 1080428.0 & 39.9456 \\
\hline
\end{tabular}

\section{Comparison with EOQ Policy}

The optimal policy obtained above yields lower cost compared with any other policy. A business manager may use the EOQ policy because it is easy to implement. However, there will be significant loss, if the optimal policy is not implemented. This significant difference in the case of the three-price problem is displayed in Table 4. The parameters considered are $K=$ Rs.2400, $F=0.2, \alpha_{1}=0.98, \alpha_{2}=0.95, P=$ Rs.100, and $D=1000$. The cost associated with the EOQ policy and optimal cost is represented in Column 2 and Column 3 of the Table. The planning horizon is considered in the first column of the Table. The percentage difference is considered in the last column. In the EOQ policy, the economic order quantity will be ordered as many times as possible, and any remaining demand is met by the remainder order. The economic order quantity 
for the first order, second order, and after the second order is given by $Q_{1}=\sqrt{\frac{2 K D}{P F}}$, $Q_{2}=\sqrt{\frac{2 K D}{P \alpha_{1} F}}$ and $Q_{3}=\sqrt{\frac{2 K D}{P \alpha_{2} F}}$. It can be observed that since the holding cost is proportional to the purchase cost, the economic order quantity for the first order will be different from the next orders. From Table 4, it can be observed that as the planning horizon increases, the percentage difference in the cost of the optimal policy and the EOQ policy decreases. This implies that the optimal policy performs better than the EOQ policy for a smaller horizon compared with a larger one.

Table 4 Comparison of Optimal Policy with the EOQ Policy

\begin{tabular}{|c|c|c|c|}
\hline$T$ & EOQ Cost (Rs.) & Optimal cost (Rs.) & Percentage difference in cost \\
\hline 1 & 110912.5 & 107751.4 & 2.9337 \\
\hline 1.5 & 163205.9 & 160023.7 & 1.9886 \\
\hline 2 & 215499.4 & 212297.1 & 1.5084 \\
\hline 2.5 & 267793.0 & 264571.1 & 1.2178 \\
\hline 3 & 320086.7 & 316845.3 & 1.0230 \\
\hline 3.5 & 372380.4 & 369119.8 & 0.8833 \\
\hline 4 & 424674.2 & 421394.4 & 0.7783 \\
\hline 4.5 & 476968.1 & 473669.0 & 0.6965 \\
\hline 5 & 529262.1 & 525943.7 & 0.6309 \\
\hline
\end{tabular}

\section{Conclusion and Suggestion for Future Research Work}

The present article considers an optimal inventory policy of the retailer in response to a special price strategy on subsequent orders by the supplier. The demand of the customers is assumed to be constant and known to the retailer. A closed form solution to the optimal number of orders and optimal order quantities are developed for the two-price problem by minimizing the total cost of the model. The two-price model is extended to the three-price problem. In order to highlight the importance of the derived optimal policy, a comparison is made with the EOQ policy. It is found that a large percentage difference exists for a smaller planning horizon. Any other similar pricing strategy of the supplier can be considered for further research work. One such similar pricing strategy is the discount only for the first purchase.

\section{References}

1. Arcelus, F.J., Pakkala, T.P.M., and Srinivasan, G. (2006). "On the interaction between retailer's inventory policies and manufacturer trade deals in response to supply-uncertainty occurrences". Annals of Operations Research, Vol. 143, pp. 45-58.

2. Arcelus, F.J., Pakkala, T.P.M., and Srinivasan, G. (2015). "Inventory replenishment for profit maximization over a finite horizon". International Journal of Operations and Quantitative Management, Vol. 21, pp. 127-139. 
3. Buzacott, J. A. (1975). "Economic order quantities with inflation". Operational Research Quarterly, Vol. 26, No. 3, pp. 553-558.

4. Erel, E. (1992). "The effect of continuous price change in the EOQ". Omega, Vol. 21, pp. 593-594.

5. Gascon, A. (1995). "On the Finite Horizon EOQ Model with cost changes". Operations Research, Vol. 43(No. 4), pp. 716-717.

6. Ghosh, A.K. (2003). "On some inventory models involving shortages under an announced”. International Journal of Systems Science, Vol. 34, No. 2, pp. 129137.

7. Goyal, S. K. (1980). "A note on the paper: An inventory model with finite horizon and price changes". Journal of the Operational Research Society, Vol. 30, No. 9, pp. 839-840.

8. Goyal, S. K. (1975). "An inventory model for a product for which price fluctuates". Operations Research, Vol. 3, No. 2, pp. 112-117.

9. Huang, W., and Kulkarni, V.G. (2003). "Optimal EOQ for announced price increases in infinite horizon". Operations Research, Vol. 51, No. 2, pp. 336-339.

10. Khouja, M., and Goyal, S. K. (2006). "Single item optimal lot sizing under continuous unit cost decrease". International Journal of Production Economics, Vol. 102, pp. 87-94.

11. Khouja, M., and Park, S. (2003). "Optimal lot sizing under continuous price decrease". Omega, Vol. 31, pp. 539-545.

12. Lev, B., and Soyster, A. L. (1979). "An inventory model with finite horizon and price changes". Journal of the Operational Research Society, Vol. 30, No. 1, pp. 43-53.

13. Lev, B., and Weiss, H.J. (1990). "Inventory models with cost changes". Operations Research, Vol. 38, No. 1, pp. 53-63.

14. Naddor, E. (1966).Inventory systems, New York: Wiley.

15. Pakkala, T.P.M., and Babitha (2013). "Study of dynamic ordering strategy under one-time discount offer". The Proceedings of the International Conference on Frontiers of Statistics and its Applications, 284-290.

16. Taleizadeh, A.A., and Pentico, D.W. (2013). "An economic order quantity model with a known price increase and partial backordering". European Journal of Operational Research, Vol. 228, No. 3, 516-525.

17. Taylor, S.G., and Bradley, C.E. (1985). "Optimal ordering strategies for announced price increases”. Operations Research, Vol. 33, No. 2, 312-325.

\section{About Our Authors}

Suresha Kharvi is currently Assistant Professor at the St. Joseph's College, Bangalore. He holds a M. Sc. in Statistics from Mangalore University. He is also research scholar in Department of Statistics, Mangalore University. He has published an article in OPSEARCH Journal.

T.P.M. Pakkala is currently Adjunct Professor in Department of Statistics, Mangalore University. He holds a Ph.D. in Statistics from Mangalore University. He has been awarded a post -doctoral research award from University of New Brunswick, Canada. $\mathrm{He}$ is a recipient of prestigious Commonwealth Fellowship for post-doctoral research 
and he carried out research at University of Exeter, England for one year. His research interests include Inventory Modelling, Data Envelopment Analysis and Stochastic Finance. His publications have appeared in several journals including Annals of Operations Research, Applied Mathematical Modelling, Computers and Operations research, Decision Sciences, European Journal of Operations Research, Journal of the Operational Research Society, Computers and Industrial Engineering, Information Systems and Operations Research, International Journal of Operations and Quantitative Management, International Journal of Production Economics, OPSEARCH and Statistical Methods. 